\title{
Plant-Derived Hematopoiesis Enhancer PG2
}

National Cancer Institute

\section{Source}

National Cancer Institute. Plant-Derived Hematopoiesis Enhancer PG2. NCI Thesaurus.

Code $C 70640$.

A proprietary botanical formulation derived from a traditional Chinese medicinal (TCM) plant with hematopoietic activity. Although the mechanism of action has yet to be fully elucidated, plant-derived hematopoiesis enhancer PG2 appears to stimulate multi-lineage progenitor cells that may be closely related to the hematopoietic stem cell. In both chemotherapy- and radiation therapy-induced animal models of myelosuppression, this agent has been shown to restore granulocyte, erythrocyte, and platelet counts to normal levels. Plant-derived hematopoiesis enhancer PG2 stimulates the production of numerous cytokines such as granulocyte-colony stimulating factor (G-CSF) and may stimulate the production of neuroendocrine hormones. 